\title{
Compétences professionnelles et transfert des apprentissages des directions d'établissement en formation universitaire : une exploration franco- manitobaine
}

Hélène Louise Archambault

Université de Saint-Boniface

Gabriel Dumouchel

Université du Québec à Chicoutimi

\section{Résumé}

Cette recherche a pour objectifs d'identifier les compétences professionnelles développées par des étudiants ayant suivi le programme de maîtrise en éducation avec spécialisation en administration scolaire de l'Université de Saint-Boniface et de distinguer les conditions facilitantes et inhibantes au transfert des apprentissages. Pour ce faire, cette recherche utilise une méthodologie qualitative centrée sur le journal de bord et l'entrevue individuelle semi-dirigée. Les résultats indiquent qu'un ensemble de compétences professionnelles 
développées par les directions d'établissement scolaire en cours de formation favoriseraient une transformation de leurs pratiques quotidiennes. La discussion souligne différentes conditions facilitant le transfert des apprentissages afin de mieux comprendre cette transformation des pratiques.

Mots-clés : formation en administration scolaire, compétences professionnelles, transfert d'apprentissage, direction d'école, réussite scolaire des élèves

\begin{abstract}
This research aims to identify the professional skills developed by students having completed the Master of Education program specializing in educational administration at Université de Saint-Boniface and to determine the conditions that facilitate or inhibit the transfer of learning. The research was conducted through a qualitative methodology centred on reflective journals and semi-structured interviews. The results indicate that the professional skill set developed by students within the educational administration program promotes change in their daily practices. Various conditions facilitating the transfer of learning are then identified to better explain this transformation.
\end{abstract}

Keywords: educational administration training, professional skills, transfer of learning, educational administration, student academic success 


\section{Introduction}

Depuis quelques décennies, les pays membres de l'Organisation de la coopération et du développement économiques (OCDE, 2017), dont le Canada fait partie, s'orientent de plus en plus vers une société axée sur le savoir (Bernatchez, 2011 ; OCDE, 2017 ; Pont et al., 2008). À cette fin, différents ministères provinciaux du Canada, comme le ministère de l'Éducation de l'Ontario (MEO, 2016), prônent l'accroissement de l'innovation, la productivité et l'entrepreneuriat. Par conséquent, pour jouer un rôle actif au sein de l'économie canadienne, de nouvelles stratégies d'éducation et de formation doivent être adoptées en vue de faciliter l'acquisition des savoirs et le développement des compétences au cours du 21e siècle. Comme l'avance Bernatchez (2011), c'est en proposant des moyens de réussir adaptés aux milieux, aux besoins et aux attentes des élèves, des enseignants et des directions d'école que l'on pourra permettre à chacun de jouer un rôle actif au sein du développement économique.

C'est dans ce contexte que le milieu scolaire traverse une vague de changements majeurs transformant ainsi le rôle et les responsabilités des directions scolaires. Pour composer avec ces transformations et les pérenniser, nous croyons, tout comme Rocque (2017), qu'il est nécessaire pour ces directions de s'engager activement dans une formation continue centrée sur la pratique. De cette façon, elles pourront développer des compétences essentielles à la poursuite de la mission éducative inscrite dans un projet collectif. C'est dans cette logique que nous avons effectué une étude empirique auprès d'étudiants en administration scolaire. Mais avant de préciser les objectifs et la méthodologie de la présente recherche, les prochaines sections déclinent d'abord son contexte et son cadre conceptuel.

\section{Contexte}

Au cours des décennies 1990 et 2000, la formation offerte aux directions d'établissement scolaire a suscité d'intenses réflexions à l'échelle internationale, provoquant ainsi des transformations majeures et des remises en question au sein de l'administration des systèmes éducatifs (D’Arrisso, 2013 ; Endrizzi et Thibert, 2012 ; Hallinger, 2011 ; Pont et al., 2008). Plusieurs pays ont alors engagé leurs systèmes éducatifs dans des réformes scolaires affectant non seulement les dimensions pédagogiques et organisationnelles 
des écoles, mais aussi le rôle et les responsabilités des directions d'établissement (Dupuis, 2004 ; Endrizzi et Thibert, 2012 ; IsaBelle et Labelle, 2017). Les nombreux changements apportés récemment au sein du milieu scolaire entraînent de nouvelles attentes à l'égard des directions d'établissement (IsaBelle et Labelle, 2017 ; Pont et al., 2008), ce qui a notamment eu pour effet de complexifier la description de tâches des directions d'établissement au fil des années (Fullan, 2015 ; IsaBelle et Labelle, 2017 ; Rocque, 2017 ; Saint-Germain, 2011). De ce fait, elles se voient constamment appelées à remplir de nouvelles fonctions, à assumer de plus en plus de nouveaux rôles, de nouvelles responsabilités et à composer avec la diversité ethnoculturelle, linguistique et religieuse (Gélinas Proulx et al., 2014). Les directions doivent aussi agir et interagir avec compétence auprès des élèves, des parents et du personnel scolaire, et ce, peu importe la situation. Pour les directions d'établissement œuvrant en milieu francophone minoritaire comme au Manitoba, on note aussi la nécessité de maîtriser la langue française, de la valoriser et de « s'engager dans le développement de la communauté francophone et d'en assurer la pérennité[, car] il est du devoir de la direction d'école de développer des stratégies afin d'inciter les élèves à aimer apprendre et vivre en français » (Leurebourg, 2013, p. 276).

Dans ce contexte de mouvance, où nous assistons à une redéfinition de la profession de direction d'école, l'acquisition de nouvelles compétences est un incontournable (Derouet et Normand, 2014). Offrir une formation basée sur des référentiels de compétences reposant sur des objectifs communs et une culture commune à tous ces professionnels demeure essentiel. Déjà en 2003, Hallinger précisait que certaines formations offertes en administration scolaire au sein des universités ne correspondaient pas aux besoins des apprenants, ce que d'autres auteurs ont également noté par la suite (Grogan et al., 2009 ; Murphy, 2006 ; Poirel et al., 2017). Dans la même veine, des chercheurs ont avancé que la formation universitaire ne semblait pas qualifier les directions d'établissement pour gérer efficacement leur école (Endrizzi et Thibert, 2012 ; Fossey et Shoho, 2006 ; Young et al., 2005). Or, pour répondre plus efficacement aux différents impératifs éducatifs, les directions d'établissement doivent désormais développer des compétences professionnelles afin d'assumer pleinement leur rôle et leurs responsabilités au sein d'un établissement scolaire aux multiples visages. Il est donc impératif d'étudier la formation destinée aux directions d'école (D'Arrisso, 2013), ce que vise la présente recherche. 
À cet effet, notre étude vise deux objectifs complémentaires. Dans un premier temps, elle cherche à mieux connaître les compétences professionnelles développées par les étudiants et les diplômés du programme de formation en éducation avec spécialisation en administration scolaire de l'Université de Saint-Boniface. Dans un second temps, notre étude vise à explorer auprès de ces mêmes personnes les conditions facilitantes et inhibantes du transfert des apprentissages vers leurs établissements scolaires.

La prochaine section présente les principaux concepts liés à notre recherche qui orienteront par la suite notre démarche méthodologique auprès des étudiants de l'établissement susnommé.

\section{Cadre conceptuel}

Depuis la fin des années 1970, plusieurs chercheurs œuvrent à identifier les caractéristiques expliquant la performance de certains établissements scolaires (school effectiveness; Teddlie et Stringfield, 2007) ainsi que ses facteurs d'amélioration (school improvement; Pelletier et al., 2015). Ce faisant, la recherche a clairement démontré que la performance d'un établissement scolaire et la réussite éducative de ses élèves passent d'abord par la qualité de l'enseignement et de son personnel de direction (IsaBelle, 2017).

Avant de s'attarder au concept du leadership, aux compétences professionnelles et au transfert des apprentissages, il importe de présenter une définition de la réussite éducative. Reconnaissant l'omniprésence de nombreuses définitions, nous avons retenu celle de Potvin et Pinard (2012), celle-ci reposant sur « une visée du développement total ou global des jeunes : soit au niveau physique, intellectuel, affectif, social, moral (spirituel)» (p. 137).

\section{Compétences professionnelles des directions d'école}

Gérer avec pertinence un établissement scolaire fait nécessairement appel à un niveau élevé de compétences professionnelles de même qu'à une conduite autonome et éthique de la part des personnes qui exercent cette fonction. De nombreuses études montrent que les directions d'école devraient posséder un ensemble de compétences professionnelles afin de contribuer significativement à la réussite éducative de tous les élèves (Anderson et al., 2013 ; IsaBelle et Labelle, 2017 ; Louis et al., 2010 ; National Policy Board 
for Educational Administration, 2008 ; Pelletier et al., 2015 ; Rocque, 2017). Ces compétences professionnelles se reflètent par des actions posées par les directions d'école réparties selon neuf domaines de pratiques ; IsaBelle et Labelle (2017) les énumèrent ainsi $^{1}$ :

1) du leadership administratif et pédagogique ; 2) d'une culture de l'école axée sur l'harmonie et la sécurité ; 3) du climat de l'établissement, axé sur la collaboration ; 4) des priorités de l'école, axées sur l'apprentissage et la réussite de tous les élèves ; 5) des attentes élevées pour tous ; 6) du suivi rigoureux des progrès des élèves et des résultats de l'établissement ; 7) de la formation et du perfectionnement professionnel des enseignants ; 8) de l'engagement des parents et de la communauté ; 9) du maintien de la performance à long terme. (p. 4)

Parallèlement, Éducation et Formation Manitoba (2014) identifie cinq domaines de connaissance et de compétence pour agir à titre de chefs de file scolaire :1) le contexte culturel et éducatif ; 2) le leadership en éducation ; 3) le leadership pédagogique ; 4) le leadership en gestion de personnel ; et 5) l'administration scolaire. Pour obtenir le brevet de leadership scolaire au Manitoba, les directions d'établissement scolaire doivent posséder une connaissance pratique des différents contextes sociaux, socioéconomiques, juridiques, politiques et culturels de l'éducation. De fait, un éventail de compétences professionnelles semble nécessaire pour permettre aux directions d'école d'assurer une gestion efficace. Précisons que ces compétences auraient une incidence sur la réussite éducative des élèves. Reconnaissant que le leadership, la pratique réflexive et le transfert des apprentissages occupent une place prépondérante dans le référentiel des compétences professionnelles essentielles aux directions d'établissement scolaire, il nous semble opportun de s'y attarder.

Le leadership. Les travaux réalisés par Marzano et al. (2005), Hallinger (2011), Sheerens (2012) et, plus récemment, par IsaBelle (2017), ont démontré que le leadership partagé exercé par les directions d'établissement scolaire avait une incidence notable sur la réussite des élèves. Précisons que le leadership partagé se définit comme «un processus d'influence, dynamique et réciproque, entre les membres d'un groupe mobilisés

1 Les neuf domaines de pratiques ne sont pas présentés ici par ordre d'importance. 
ensemble pour atteindre une même cible » (Luc et Le Saget, 2013, p. 11). En effet, par leur façon de gérer, les directions joueraient un rôle déterminant quant à la performance scolaire des élèves en agissant sur la motivation et les capacités des enseignants ainsi que sur l'environnement scolaire (Lapointe et Gauthier, 2005 ; Louis et al., 2010 ; Pont et al., 2008 ; Rocque, 2017). Par ce leadership, elles établissent des alliances entre l'école et la collectivité, favorisant ainsi la collaboration afin de mieux répondre aux besoins diversifiés des élèves. Elles assurent un enseignement et un apprentissage efficaces par des stratégies visant à soutenir l'ensemble de son personnel. Ainsi, pour favoriser la réussite éducative et le mieux-être de tous les élèves, les directions d'établissement doivent mobiliser et diriger leur personnel scolaire et de soutien. De plus, elles doivent gérer efficacement les ressources matérielles et financières en préparant des états financiers selon les règles budgétaires, les normes établies et le respect des orientations de l'institution selon les politiques gouvernementales manitobaines (Éducation et Formation Manitoba, 2014). Enfin, pour soutenir, guider et mobiliser l'équipe-école, les directions d'établissement doivent poser diverses actions concomitantes afin d'assurer, de stimuler ou d'enrichir les activités qui conduisent à la réussite de tous les élèves. Par exemple, celles-ci seront en mesure de prendre une position décentralisée tout en partageant le pouvoir décisionnel avec son personnel, et de favoriser une culture de collaboration entre les enseignants, les parents, les élèves et la communauté tout en respectant les spécificités de son milieu afin de promouvoir la réussite de tous les élèves.

La pratique réflexive. Une démarche de pratique réflexive pour la construction du savoir professionnel est une compétence incontournable pour les directions d'établissement, car « la réflexion sur l'action les amène à se doter d'une épistémologie de l'agir professionnel » (Guertin, 2012, p. 77). La pratique réflexive décrite par Schön (1994), à savoir « une forme de conversation avec une situation » (p. 349), représente « une démarche d'introspection qui conduit au savoir dans l'action » (Guertin, 2012, p. 77). Selon Le Boterf (2015), cette capacité d'auto-analyse constitue une compétence essentielle à un agir professionnel compétent. Nous pouvons ainsi avancer qu'une direction d'établissement scolaire compétente « doit donc avoir la faculté de se distancier. Aussi bien par rapport à la situation que par rapport à ses pratiques » (Le Boterf, 2015, p. 133), ce qui constitue la réflexivité qui se traduit par une posture de prise de recul de la part du praticien. 
Le transfert des apprentissages. Diriger une école au 21e siècle requiert l'implantation d'un cadre de travail cohérent répondant aux différentes exigences formulées par la société ainsi qu'aux aspirations des apprenants (Girard, 2018). En 2010, Dweck suggérait, dans ses travaux sur la mentalité de croissance des directions d'établissement, une perception dynamique des compétences personnelles et professionnelles, les considérant comme continuellement à parfaire. Pour la direction, Girard précise que « la mentalité de croissance est particulièrement importante puisqu'elle permet, à la direction comme pour tout le monde, de veiller à l'actualisation des connaissances et des pratiques » $(2018$, section « ...et pour la direction? »). Déjà en 2011, Bernatchez notait que les directions d'établissement s'investissaient dans des démarches de formation continue et d'apprentissage, ce qui les amenait à développer et à rehausser leurs compétences professionnelles tout en renforçant leurs pratiques de transfert des connaissances. À l'instar de Tardif et Meirieu (1996), nous sommes d'avis que la formation offerte au sein de «l'institution scolaire peut grandement influer sur le transfert des connaissances » (p. 4). Roussel (2011) définit le transfert « comme étant l'utilisation, par un individu, des connaissances, des savoirs et des habiletés appris en formation dans le cadre de contextes de travail comportant un certain degré de nouveauté, et ce, afin d'améliorer, de façon prioritaire sa performance » (p. 30). Pour Tardif et Meirieu (1996), le transfert « requiert des interventions plurielles et non singulières [... et d]e nombreux facteurs doivent être pris en considération de façon concomitante pour influer sur le transfert des connaissances » (p. 9). Pour favoriser ledit transfert, Roussel (2011) recommande une démarche de formation où le processus de transfert est composé de trois temps en alternance, soit la contextualisation (application de connaissances directement liées à un contexte donné), la décontextualisation (dégagement de principes, de modèles, des façons de faire applicables à divers contextes de transfert) et la recontextualisation (applications des connaissances acquises à de nouvelles situations). C'est par la mise en place de dispositifs de formation basés sur « une approche dialogique et interprétative, sur différentes étapes de conscientisation, de distanciation et de recul réflexif » (Eneau et al., 2012, p. 4) que la direction d'établissement pourra viser le transfert de ses apprentissages vers de nouvelles situations en milieu scolaire.

Les éléments contextuels et conceptuels de notre recherche ayant été présentés et argumentés, la prochaine section précisera la méthodologie employée pour réaliser la présente étude. 


\section{Méthodologie}

Cette recherche s'inscrit principalement dans le paradigme qualitatif/interprétatif. Comme le souligne Savoie-Zajc (2018), l'objectif de ce courant est principalement « de mieux comprendre le sens qu'une personne donne à son expérience » (p. 192). Cette recherche se concentre davantage « sur l'analyse des processus sociaux, sur le sens que les personnes et les collectivités donnent à l'action, sur la vie quotidienne, sur la construction de la réalité sociale » (Deslauriers, 1991, p. 6). Ainsi, ce type de recherche peut être visualisé comme un système d'activités humaines favorisant l'émergence d'un processus de collaboration entre le chercheur et les participants (Manning, 1997 ; SavoieZajc, 2018).

\section{Participants}

Issu de différents milieux éducatifs, notre échantillon est composé de 21 participants francophones, soit 12 femmes et 9 hommes âgés de 31 à 61 ans. Les participants devaient répondre aux critères suivants :

- $\quad$ Etre francophone

- $\quad$ Etre d'âge adulte (18 ans et plus)

- Agir en tant que personnel de direction dans une école ou dans un autre milieu de pratique lié au système éducatif

- Appartenir à l'une des quatre catégories suivantes :

- Être inscrit à titre d'étudiant ou étudiante au programme de maîtrise en éducation avec spécialisation en administration scolaire de l'Université de Saint-Boniface (formation en présentiel ou en ligne)

- Être un étudiant diplômé ou étudiante diplômée du programme de maîtrise en éducation avec spécialisation en administration scolaire de l'Université de Saint-Boniface (formation en présentiel ou en ligne)

- Être un étudiant diplômé ou étudiante diplômée d'un programme de maîtrise en éducation avec une spécialisation autre qu'en administration scolaire de l'Université de Saint-Boniface (counseling, langue et littératie, éducation inclusive) (formation en présentiel ou en ligne) 
○ Être une ressource professorale au sein du programme de maîtrise en éducation avec spécialisation en administration scolaire de l’Université de Saint-Boniface (formation en présentiel ou en ligne)

La chercheuse principale, à partir d'informations reçues de différents contacts œuvrant en contexte scolaire, a envoyé une lettre d'invitation par messagerie électronique aux participants qu'elle désirait recruter. La majorité des participants proviennent du Manitoba $(n=18)$ et trois participants demeurent hors de cette province, soit en Colombie-Britannique $(n=1)$, en Ontario $(n=1)$ et au Québec $(n=1)$. Soulignons que les participants font partie du personnel de direction dans une école ou dans un autre milieu de pratique lié au système éducatif. Ils œuvrent au sein d'établissements scolaires de niveau primaire, intermédiaire, secondaire, collégial ou universitaire.

\section{Outil de collecte de données}

En nous inspirant des travaux de Bertaux (1976) et de Desgagné (2005) portant sur les récits exemplaires de pratique enseignante, nous avons, dans un premier temps, privilégié un échange collaboratif sous forme d'entrevue individuelle semi-dirigée composée de trois parties : 1) un récit de réussite (un succès vécu);2) un récit de pratique d'exploration (une difficulté rencontrée); et 3) le transfert des apprentissages. Audet (2003) souligne que la narration de récits de pratique permet « l'accès à l'action et au "sens" donné à cette action, donc à la logique de l'acteur » (p. 121) telle qu'il l'a vécue. C'est donc à partir de cette narration des récits de pratique que nous avons relevé les compétences professionnelles développées dans le cadre du programme de formation en administration scolaire. Précisons que le canevas du récit de réussiteet celui du récit d'exploration étaient identiques pour tous les groupes de participants. Pour ce qui est de la partie de l'entrevue portant sur le transfert des apprentissages, nous avons développé quatre protocoles d'entrevue distincts en fonction de chaque groupe de participants. Grâce à cette narration, les étudiants (acteurs-praticiens), les diplômés (anciens étudiants) ayant suivi la formation en présentiel ou en ligne, les autres gestionnaires scolaires et les ressources professorales ont pu mettre en lumière leurs réflexions quant aux conditions (leviers et freins) du transfert des apprentissages vers les terrains professionnels concernés, soit les établissements scolaires ou autres lieux de pratique connexes. 
Dans un second temps, nous avons consigné, dans un journal de bord, des notes descriptives, méthodologiques, théoriques et personnelles tout au long de notre démarche de recherche (Baribeau, 2005). Pour Savoie-Zajc (2018), un tel journal de bord remplit trois fonctions : «garder le chercheur en état de réflexion active pendant sa recherche, lui fournir un espace pour exprimer ses interrogations, ses prises de conscience, et consigner les informations qu'il juge pertinentes » (p. 213). C'est ainsi que, dès le début et tout au long de notre démarche, nous avons consigné de telles notes dans un journal de bord selon une forme spontanée (Baribeau, 2005 ; Savoie-Zajc, 2018).

\section{Déroulement}

Du 1er février au 8 mars 2016, des entrevues semi-dirigées ont été réalisées avec tous les participants, in situ ou par Skype, dans leur milieu de travail. Précisons que nous avons utilisé la plateforme technologique Skype, pour contrer la distance avec les participants résidant à l'extérieur du Manitoba, de même que iFree Skype recorder, pour l'enregistrement audio des entrevues. Toutes les entrevues enregistrées ont été transcrites à des fins d'analyse.

\section{Analyse des données}

Pour cette recherche, les données récoltées ont fait l'objet d'une analyse de contenu. Cette méthode vise la codification des éléments du matériel récolté de manière à permettre au chercheur de mieux en connaître les caractéristiques et la signification (L'Écuyer, 1990). Une première lecture de la transcription des entretiens nous a permis d'identifier les thèmes émergents, soit les catégories déterminées en fonction des principaux thèmes abordés par les participants. Par la suite, pour chacune des catégories retenues, des catégories de second niveau hiérarchique ont été identifiées de façon inductive. Cette démarche nous a permis de créer, puis de valider une liste de codes qui a par la suite servi à analyser l'ensemble du matériel de notre étude (Miles et Huberman, 2003). Nous avons ensuite fait ressortir les éléments significatifs des écrits, des discours et des observations consignées dans le journal de bord de la chercheuse principale pour l'ensemble des participants, et ce, pour chacun de nos objectifs de recherche.

Quant à la sélection des propos pertinents émis par les répondants, elle s'est faite, d'une part, en fonction de l'éclairage que ces derniers pouvaient apporter au 
développement et au perfectionnement des compétences professionnelles. D'autre part, cette sélection s'est réalisée selon la compréhension des conditions sous-jacentes au transfert des apprentissages opéré par les étudiants inscrits et les diplômés qui œuvrent dans les milieux scolaires ou au sein d'un autre milieu de pratique lié au système éducatif. Il importe de souligner que nous avons respecté le discours original sur le plan linguistique émis par les répondants lors des entrevues en veillant à préserver l'anonymat des participants.

\section{Résultats}

Dans cette partie, nous présentons les principaux résultats qui ont émergé des outils de la collecte de données. Pour chaque objectif, nous ressortons les éléments significatifs des écrits, des discours et des observations consignées dans le journal de bord de la chercheuse en fonction des quatre groupes de répondants de l'étude auxquels nous avons assigné des codes précis dans le cadre de cet article : (1) les étudiants (acteurs-praticiens) inscrits à la formation de deuxième cycle au programme de maîtrise en éducation avec spécialisation en administration scolaire de l'Université de Saint-Boniface [EI] ; (2) les diplômés (anciens étudiants) ayant suivi la formation [ED] ; (3) les étudiants diplômés d'autres formations (ou autres gestionnaires scolaires) [EDA] ; de même que (4) les ressources professorales impliquées [RP].

\section{Identification des compétences professionnelles}

Pour rappel, le premier objectif visait à identifier les compétences professionnelles développées par les étudiants et les diplômés du programme de formation en éducation avec spécialisation en administration scolaire de l'Université de Saint-Boniface.

Le profil des compétences développées. Lors des entrevues individuelles semidirigées, la majorité des participants $(n=18)$ ont mentionné que plusieurs compétences développées lors de leur formation sont appliquées régulièrement dans leurs pratiques de leader au sein de leur établissement scolaire. À titre d'exemple, un participant reconnaît que la formation lui a permis de « savoir approcher les choses, savoir approcher les personnes, savoir établir une communication efficace avec l'autre, savoir être à l'écoute 
empathique et active, savoir gérer son stress, être un leader » $\left(\right.$ TAEI13) ${ }^{2}$. En matière de leadership partagé, nous retenons divers propos, dont celui-ci :

C'est ma philosophie, [...] un leadership où je sers les gens, je suis là pour les aider et les appuyer. Certainement, je crois que le leadership partagé est la clé pour assurer les gens, qu'ils sachent qu'ils ont leur mot à dire et que leur mot va être écouté et pris au sérieux. (RPAED14)

Un autre participant déclare : « il faut avoir un leadership partagé, il faut avoir confiance au personnel afin d'avoir une école qui est efficace » (RPARP03). Une étudiante inscrite au programme de formation en administration mentionne : « Je pense qu'aussitôt que tu as un leadership partagé, tu as une gestion efficace » (RPEEI16). Une diplômée souligne quant à elle que la formation lui a permis de développer « le leadership partagé, comment influencer la résolution de problèmes, analyser, trouver d'autres options, penser hors de la boîte. Et, tout cela fait partie de mes études » (TAED12).

En lien avec les compétences à acquérir, les participants citent régulièrement la pratique réflexive (analyser, réfléchir et la prise de recul) comme un moyen pédagogique susceptible de favoriser le développement des compétences professionnelles. En effet, une ressource professorale note que « c'est vraiment une priorité de développer chez nos futurs leaders, la pratique réflexive » (TARP06). De son côté, une étudiante déclare : « je pense que je réfléchis toujours à mes pratiques. Des fois, je me demande qu'est-ce que je fais et pourquoi je le fais » (TAEI16). Pour sa part, une diplômée œuvrant comme direction d'école mentionne l'importance que revêtent l'analyse et la réflexion de ses pratiques professionnelles dans le cadre des cours offerts en administration scolaire :

Bien, les réflexions dans les cours, on est souvent demandé de faire des réflexions et c'est cela, c'est d'être capable d'analyser, et je pense que je suis plus compétente à analyser la situation et de voir bon, comment on pourrait avoir cette

2 Signification des acronymes : les acronymes sont formés de trois éléments, le premier constitue l'endroit d'où est tiré la citation $(\mathrm{TA}=$ Transfert des apprentissages $/ \mathrm{RPA}=$ Récit de pratique accomplissement $/ \mathrm{RPE}=\mathrm{Récit} \mathrm{de}$ pratique exploration) ; le second, le type de participant $(\mathrm{ED}=$ Étudiant diplômé / EDA = Étudiant diplômé autre formation / EI = Étudiant inscrit / RP =Ressource professorale); et finalement, le nombre final correspond au numéro de participant. Ainsi, TAEI13 signifie « Transfert des apprentissages étudiant inscrit numéro 13 ». 
option, cette solution, cette option-là, mais laquelle va amener à l'efficacité ou cela va aider la situation. (TAED12)

Une diplômée qui œuvre au sein d'une équipe pluridisciplinaire offrant des services aux élèves ajoute : «Je dirais que souvent il faut prendre une prise de recul et analyser ce que l'on sait, ce que l'on a appris et ce que l'on veut pour notre école » (TAED11). Enfin, soucieux de la réussite scolaire des élèves fréquentant son école, un étudiant inscrit au programme de formation en administration scolaire et œuvrant à titre de directeur d'école déclare : « qu'il faut prendre ce temps de recul. Qu'est-ce que 1'on est en train de faire ? Est-ce que c'est efficace ? Est-ce que cela aide les enfants ? À la fin de la journée, tu es censé aider tous les élèves » (TAED12). Les observations inscrites au journal de bord soulignent l'importance pour les participants de travailler en équipe tout en ayant une vision commune quant à la réussite de tous les élèves.

\section{Transfert des apprentissages}

Le deuxième objectif de notre recherche visait à explorer les conditions facilitantes et inhibantes au transfert des apprentissages par les étudiants et les diplômés du programme de formation en éducation avec spécialisation en administration scolaire de l'Université de Saint-Boniface.

Des conditions facilitant le transfert des apprentissages. En analysant les données recueillies au cours des entretiens, nous avons identifié différentes conditions facilitant le transfert des apprentissages. Nous constatons ainsi que la majorité des participants ont précisé que l'accessibilité et l'appui offert par le corps professoral lors de la formation avaient une incidence sur le transfert des apprentissages. À ce sujet, un diplômé du programme de formation en counseling, agissant à titre d'adjoint à la direction d'une école offrant des classes de la maternelle à la douzième année, a affirmé que « L'accessibilité des enseignants, du corps professoral, des profs qui répondent à leurs courriels, à leurs appels, qui fournissent de l'appui individualisé au besoin, favorise le transfert des apprentissages » (TAEDA01). Dans la même foulée, un professeur enseignant au sein du programme de formation souligne que la simulation d'une situation donnée à l'intérieur de la formation contribue à la transférabilité des apprentissages, car « on fait du pratico-pratique pendant la formation. Alors, c'est des jeux de rôle, 
des occasions où les gens peuvent simuler et mettre en pratique » (TARP02) les apprentissages réalisés lors de la formation. De son côté, un diplômé déclare : « Je sais que j'ai suivi plusieurs cours avec [nom du professeur], on faisait des jeux de rôle dans la classe, cela aussi c'est important, cela a favorisé la consolidation de mes connaissances » (TAEDA01). Une diplômée œuvrant à titre de directrice d'école précise quant à elle que les cours suivis lui ont permis « de réfléchir, de mettre en pratique ce que l'on pense avoir appris, de discuter, de pouvoir poser des questions, entendre des invités parler et de comparer ce qu'ils font » (TAED05). Un autre étudiant ajoute que la transférabilité des apprentissages passe nécessairement par « la discussion et les travaux qui sont demandés aux étudiants. Je pense que ça devrait toujours être conçu pour faire un lien avec la pratique sur le terrain » (TAEI08). Une enseignante qui a la possibilité de remplacer la direction d'école lorsque celle-ci s'absente déclare que « les conditions facilitantes [...] seraient quand j'ai la chance de faire l'enseignante désignée, parce que cela me donne des opportunités de mettre en pratique qu'est-ce que j'apprends dans mes cours. Ce qui est super! » (TAEI16). Un directeur d'école inscrit au programme de formation reconnaît toute la valeur de réaliser la formation alors qu'il est dans un poste de direction d'école : «Quand tu fais ta maîtrise, tu occupes le poste [de direction d'école]. Dans mon cas, je suis déjà directeur d'école, je ne suis pas seulement dans la théorie, je suis aussi dans la pratique, les deux se nourrissent très bien! » (TAEI17). Enfin, soulignons qu'une ressource professorale abonde dans le même sens en précisant que « le transfert est beaucoup plus facile pour les personnes qui sont déjà en direction que pour les autres qui sont encore en classe [comme enseignant] » (TARP03).

Des conditions inhibant le transfert des apprentissages. Par ailleurs, l'analyse des résultats nous a permis d'identifier trois principales conditions qui limitent le transfert des apprentissages, soit le manque de liens entre la théorie et la pratique lors de la formation, l'authenticité des travaux demandés par le corps professoral de même que la pertinence des rétroactions émises par ce dernier. De ce fait, au cours de la collecte de données, plusieurs étudiants inscrits et diplômés ont clairement énoncé que l'application de la théorie à la pratique quotidienne est capitale pour favoriser le transfert des apprentissages en milieu scolaire. Une minorité d'étudiants ont cependant souligné que cette application de la théorie à la pratique sur le terrain n'est pas toujours évidente. À ce sujet, un étudiant déclare : 
Il n'y a pas de liens avec la pratique sur le terrain. Souvent, la matière elle-même fait des liens avec la pratique de façon générale, mais quand on pense à ma pratique à moi, c'est juste dans les travaux que je le fais, c'est dans les réflexions que je le fais et puis c'est ça qui doit être encouragé par les profs. (TAEI08)

Une autre étudiante précise que « si par la théorie ou les exercices demandés on ne voit pas les liens avec notre pratique, pour moi, c'est un obstacle au transfert des apprentissages » (TAEDA04).

Notons également que certains étudiants ont déploré le fait que quelques évaluations demandées dans le cadre de la formation universitaire ne contribuent pas nécessairement à rehausser leurs compétences professionnelles puisque l'accent est davantage mis sur le produit final que sur le processus d'apprentissage. À ce propos, un étudiant inscrit au programme de formation œuvrant en direction d'école a noté :

J'ai eu un autre cours, c'était probablement le devoir le plus frustrant, on était cinq [étudiants] dans le cours et [...] personne avait compris ce que l'on devait faire, ça fait qu'on a dû le recommencer. C'était un travail ardu, c'était pas comment ça va m'aider [dans ma pratique], c'était comment que je vais répondre aux exigences du prof, ça je hais ça comme la peste. (TAEI17)

Pour sa part, un étudiant inscrit au programme de formation affirme que certaines rétroactions émises par le corps professoral sont contre-productives et non pertinentes pour susciter la pratique réflexive :

Il y avait des rétroactions que je trouvais contre-productives... ou je devais faire des commentaires de 255 mots ou 300 mots, et puis, voilà, il faut refaire votre travail parce qu'il contient 25 mots de trop, il faut être concis, précis. Je préfère qu'il m'enlève le point et que ma conscience elle soit tranquille, comment je vais dire, j'ai une analyse qui me satisfait. Je n'étais pas pour donner une réponse pour donner une réponse [...]. Donc, pour cela, je trouve que c'est contre-productif. (TAEI13) 


\section{Discussion}

L'objectif premier de cette étude était d'identifier les compétences professionnelles développées par les étudiants ayant suivi le programme de maitrise en éducation avec spécialisation en administration scolaire de l'Université de Saint-Boniface. Comme d'autres chercheurs avant nous (Dupuis, 2004 ; IsaBelle et Labelle, 2017 ; Pelletier et al., 2015 ; Pont et al., 2008 ; Rocque, 2017 ; Sackney, 2007), nous constatons que les directions d'école identifient plusieurs compétences à acquérir pour favoriser la réussite scolaire des élèves. Une étude réalisée par Hord et Sommers (2008, cité dans IsaBelle et al., 2013) démontre que «l'écoute, le questionnement, le sens de l'observation, l'ouverture d'esprit, la connaissance des objectifs, la réflexion dans l'action, le jugement professionnel, la confiance et, bien sûr, le leadership » (IsaBelle et al., 2013, p. 159160) sont des compétences essentielles pour œuvrer en direction d'école. Il est donc encourageant de constater que la majorité des participants à notre étude révèlent avoir développé ces compétences professionnelles en cours de formation.

Dans un autre ordre d'idées, notons que la pratique réflexive ressort comme un incontournable, voire une nécessité dans les programmes de formation offerts aux directions d'établissement scolaire (Lison, 2013). Pour Frenay et Bédard (2004), la capacité réflexive des étudiants s'avère une condition essentielle pour favoriser le transfert des apprentissages. Rappelons que « le praticien réflexif est centré sur l'action mais son but est de comprendre, d'extraire du savoir à propos de ses propres réflexions. Il dialogue sur ses situations pour les comparer, les analyser, les mettre en question, les enrichir » (Donnay, 2006, cité dans Biémar et al., 2008, p. 74). Soulignons que de nombreux participants reconnaissent l'analyse, la réflexion et la prise de recul, omniprésentes dans la formation, comme essentielles à la transformation efficiente et efficace de leurs pratiques. D'un point de vue épistémologique, cette démarche réflexive et critique permet aux gestionnaires de s'interroger sur le bien-fondé de leurs pratiques tout en les rendant plus aptes à influencer les différents acteurs scolaires et de la communauté sur le plan des valeurs, de l'éthique professionnelle, de la vision et de la mission de l'école.

À titre de rappel, le second objectif de notre étude concernait l'exploration des conditions facilitantes et inhibantes du transfert des apprentissages vers les établissements scolaires. En ce qui concerne les conditions qui facilitent la transférabilité 
des connaissances et des habiletés acquises lors de formations dans la pratique, les participants rencontrés soulignent que l'accessibilité, l'appui du corps professoral, l'apprentissage contextualisé et le fait d'être un apprenant en poste à la direction d'école lors de la formation sont des facteurs ayant une incidence positive sur le transfert des apprentissages. De ce fait, nous croyons que ces différents facteurs touchent directement la construction des connaissances, des compétences individuelles et collectives, du développement des habiletés et du transfert dans la pratique réelle de l'apprenant.

Par ailleurs, nos résultats mettent également en évidence quelques conditions inhibantes au transfert. De fait, pour quelques participants, il semble difficile de transférer les apprentissages acquis lorsque les liens entre la théorie et la pratique ne sont pas évidents et que les activités pédagogiques et évaluatives ne sont pas signifiantes et authentiques. À ce propos, la liaison entre la théorie et la pratique doit être centrée sur des applications concrètes tirées du terrain où des discussions authentiques avec des pairs et des mentors, de l'observation, de la critique, de la pratique réflexive, ainsi que des rétroactions constructives de la part de professionnels experts ont leur place. Nous croyons que les situations d'apprentissage et d'évaluation doivent s'effectuer en fonction de la pratique réelle de l'apprenant.

À l'instar de Frenay et Bédard (2004) de même que Roussel (2011), nous avançons que le choix des activités pédagogiques proposées par les formateurs (corps professoral) aux étudiants est déterminant pour favoriser la transférabilité des apprentissages. En effet, de l'avis de la majorité des participants rencontrés, les formateurs ont su proposer des situations d'apprentissage signifiantes et authentiques qui favorisaient l'acquisition de connaissances et d'habiletés utiles afin qu'il y ait un transfert des apprentissages dans des tâches courantes de leur pratique professionnelle au sein de leur établissement scolaire. De plus, il nous apparaît capital de préciser que le corps professoral rencontré dans le cadre de cette recherche est activement impliqué dans la recherche d'activités pédagogiques signifiantes en vue de susciter, voire provoquer la capacité réflexive facilitant ainsi le transfert des apprentissages des étudiants en administration scolaire.

En somme, notre étude démontre que le transfert des apprentissages en contexte scolaire semble dépendre de diverses conditions associées « à l'environnement professionnel du participant et au participant lui-même [tout comme] aux dispositifs pédagogiques de la formation » (Petit et al., 2012, p. 55). En s'inspirant des travaux 
de Mintzberg (2012), il est possible d'affirmer que la transformation des pratiques des directions d'établissement scolaire passe inévitablement par une interaction entre la réflexion, l'analyse, l'action, l'expérience et la collaboration, des savoirs constituant les bases du leadership axé sur une vision partagée.

\section{Conclusion}

Diriger un établissement scolaire demeure une affaire complexe nécessitant l'acquisition et le perfectionnement de compétences professionnelles variées. Pour un savoir-agir compétent de tout personnel exerçant une fonction d'encadrement au sein d'une école, plusieurs indices du discours des participants indiquent la présence de compétences clés. Ces compétences peuvent consister en la pratique d'un leadership partagé, la capacité de gérer stratégiquement les ressources humaines, la mobilisation du personnel et le développement éducatif dans une perspective de réussite pour tous les élèves. En fait, les résultats de cette recherche démontrent que les directions d'établissement scolaire doivent posséder des compétences essentielles pour gérer les ressources et orienter les pédagogies et l'apprentissage en vue d'une amélioration du rendement scolaire de tous les élèves. Par ailleurs, les données recueillies ont permis d'identifier différentes conditions facilitantes et inhibantes au transfert des apprentissages des apprenants.

Former les citoyens de demain nécessite la mise en place d'un leadership partagé et d'une culture de collaboration axée sur la réussite scolaire de tous les élèves. Les directions d'établissement scolaire doivent offrir des possibilités d'entraide, de support mutuel et de collaboration entre élèves, enseignants, parents et communautés. En faisant appel aux compétences de chacun, on encourage les acteurs à s'entraider, à collaborer et à coopérer lors de projets éducatifs qui promeuvent la réussite éducative des élèves.

Travailler au succès scolaire de tous les élèves, c'est donc regarder plus étroitement la formation universitaire en administration scolaire offerte au sein des universités. De fait, cette formation est amarrée directement à la pratique professionnelle puisqu'elle est visiblement responsable de l'adoption de diverses modalités d'apprentissage permettant le développement de compétences professionnelles et le transfert des apprentissages des directions d'établissement scolaire actuelles et futures. Cela dit, pour valoriser le transfert des apprentissages vers les milieux de pratique, il 
serait bénéfique d'offrir un stage à l'intérieur du programme de formation, comme l'exige la province manitobaine pour le brevet en leadership. Cela permettrait à l'étudiant qui n'occupe pas un poste de direction de « vivre une expérience terrain » rattachée plus spécifiquement aux fonctions associées à la gestion scolaire et au leadership en éducation. Grâce à cette expérience pratique, l'apprenant pourrait donc appliquer les différentes notions théoriques acquises lors de la formation dans un contexte scolaire particulier.

Par ailleurs, nous tenons à souligner que cette recherche est particulièrement novatrice pour l'Université de Saint-Boniface puisqu'à notre connaissance, aucune étude du programme en administration scolaire n'avait été réalisée depuis son implantation en 1982. Grâce à cette recherche, nous disposons désormais de données probantes pour ce programme universitaire. À la lumière des résultats obtenus, nous pouvons démontrer qu'il permet aux membres du personnel œuvrant dans les écoles et les divisions scolaires de la province manitobaine de développer un savoir-agir adapté à leurs contextes marqués par des réalités sociales spécifiques.

Il existe quelques limites inhérentes à la recherche qualitative que nous avons menée (Deslauriers et Kérisit, 1997). Tout d'abord, la procédure d'échantillonnage dite « non probabiliste » limite la généralisation des résultats à la population compte tenu du nombre restreint de participants à cette recherche. Les résultats présentés reposent sur ce qu'on appelle des données autorapportées ; ce faisant, ces derniers se limitent uniquement aux acteurs concernés. Considérant que plusieurs participants connaissaient la chercheuse principale, il est possible que ces derniers aient pu être influencés par le phénomène de la désirabilité sociale (Tournois et al., 2000). De plus, rappelons que cette recherche a été réalisée dans une seule université, ce qui limite aussi la généralisation des résultats. Nous croyons toutefois que notre étude a permis de cerner la contribution de cette formation à la pratique du leadership en contexte scolaire francophone en milieu minoritaire. À la lumière des résultats obtenus, nous pouvons prudemment avancer que les directions d'établissement acquièrent des qualités de leadership ayant possiblement une incidence positive sur le rendement et le bien-être des élèves. Cette étude permet également d'avoir une meilleure compréhension de l'application des cinq domaines de connaissance et de compétence en matière de leadership scolaire selon le guide des exigences pour le brevet de leadership scolaire du Manitoba (Éducation et Formation Manitoba, 2014). Elle nous amène aussi à mieux comprendre, de façon plus concrète, en quoi consiste le transfert des compétences en matière de leadership scolaire. Enfin, cette recherche a permis de 
proposer quelques indicateurs en matière de développement professionnel dans une optique de savoir-agir avec compétence.

Quelques perspectives de recherches futures peuvent découler des résultats obtenus. Reconnaissant que les défis sont nombreux et variés en contexte scolaire, il s'avère important d'amorcer de nouvelles études portant sur les caractéristiques individuelles des directions d'établissement, à savoir leurs attitudes, leurs croyances et leurs perceptions quant à la dynamique du transfert des apprentissages dans leur pratique professionnelle. Comprendre l'apport des différents facteurs individuels, organisationnels, institutionnels ou sociétaux dans le transfert des acquis lors de formations sur les terrains de pratique professionnelle des directions d'établissement nécessite aussi la réalisation de futurs travaux de recherche. Puisque les programmes de formation universitaire ont un rôle prépondérant dans la construction des compétences professionnelles et le transfert des apprentissages des directions d'établissement, il serait fort pertinent de mieux comprendre la structure du processus de transfert lors du déroulement de la formation offerte dans le cadre de programmes de maîtrise en éducation en administration scolaire en milieu universitaire. De plus, il nous semblerait aussi important d'explorer le transfert des apprentissages de manière longitudinale afin de mieux comprendre l'alliance entre la théorie et la pratique dans ledit domaine de formation.

Finalement, nous souhaitons que cette étude permette aux directions d'établissement scolaire de porter un regard critique sur leurs pratiques, leurs compétences professionnelles et le transfert de leurs apprentissages en matière de leadership partagé afin de progresser professionnellement dans un processus d'amélioration continue au service du système éducatif. 


\section{Références}

Anderson, S. E., Rodway-Macri, J., Yashkina, A. et Bramwell, D. (2013, mai). Characteristics of elementary schools achieving consistently high or low percentages of students at the provincial standard on EQAO Assessments. https:// www.oise.utoronto.ca/cidec/UserFiles/File/Research/Final_Report_EQAO School Success Study June 111.pdf

Audet, G. (2003). Récits de pratique et formation à la diversité culturelle. Dans M. Abdallah-Pretceille (dir.), Former et éduquer en contexte hétérogène. Pour un humanisme du divers (p. 110-127). Anthropos.

Baribeau, C. (2005). Le journal de bord du chercheur. Recherches Qualitatives, HorsSérie(2), 98-114. http://www.recherche-qualitative.qc.ca/documents/files/revue/ hors serie/hors serie v2/CBaribeau\%20HS2-issn.pdf

Bernatchez, J. (2011). La formation des directions d'établissement scolaire au Québec : apprendre à développer un savoir-agir complexe. Télescope, 17(3), 158-175. https://telescope.enap.ca/Telescope/docs/Index/Vol_17 no 3/Telv17n3 bernatchez.pdf

Bertaux, D. (1976). Histoires de vies ou récits de pratiques? Méthodologie de l'approche biographique en sociologie. Maison des sciences de l'homme.

Biémar, S. Dejean, K. et Donnay, J. (2008). Co-construire des savoirs et se développer mutuellement entre chercheurs et praticiens. Recherche et formation, (58), 71-84. http://rechercheformation.revues.org/722

D’Arrisso, D. (2013). Pressions et stratégies dans la formation professionnelle universitaire : Le cas de la formation des directions d'établissement scolaire du Québec (1988-1989 à 2008-2009) [Thèse de doctorat]. Université de Montréal. https://papyrus.bib.umontreal.ca/xmlui/handle/1866/10219

Derouet, J.-L. et Normand, R. (2014). La question du leadership dans la tradition française : de la communauté scolaire au management. Dans J.-L. Derouet et R. Normand (dir.), La question du leadership en éducation : Perspectives européennes (p. 41-63). L’Harmattan. 
Desgagné, S. (2005). Récits exemplaires de pratique enseignante : analyse typologique. Presses de l'Université du Québec.

Deslauriers, J.-P. (1991). Recherche qualitative : guide pratique. McGraw-Hill.

Deslauriers, J.-P. et Kérisit, M. (1997). Le devis de recherche qualitative. Dans J. Poupart, J.-P. Deslauriers, L. H. Groulx, A. Lapierre, R. Mayer et A. P. Pires (dir.), La recherche qualitative : Enjeux épistémologiques et méthodologiques (p. 85-112). Gaëtan Morin.

Dupuis, P. (2004). L'administration de l'éducation : quelles compétences ? Éducation et Francophonie, 32(2), 133-157. https://www.acelf.ca/c/revue/pdf/ Ladministrationdeleducation.pdf

Dweck, C. S. (2010). Mind-sets and equitable education. Principal Leadership, 10(5), 26-29. https://eschs.weebly.com/uploads/2/5/1/7/25174886/mind-sets-andequitable-education.pdf

Éducation et Formation Manitoba. (2014, décembre). Guide des exigences pour le brevet de leadership scolaire. https://www.edu.gov.mb.ca/m12/perfprof/brevet/docs/ leadership.pdf

Endrizzi, L. et Thibert, R. (2012). Quels leaderships pour la réussite de tous les élèves ? Dossier d'actualités Veille et analyses, (73), 1-28. https://www.researchgate.net/ publication/264991711_Quels_leaderships_pour_la_reussite_de tous_les_eleves

Eneau, J., Bertrand, E. et Lameul, G. (2012). Se former et se transformer : perspective critique et formation universitaire aux métiers de la formation. Revue internationale de pédagogie de l'enseignement supérieur, 28(1), 1-12. http:// ripes.revues.org/585

Fossey, R. et Shoho, A. (2006). Educational leadership preparation programs: In transition or in crisis? Journal of Cases in Educational Leadership, 9(3), 3-11. https://doi.org/10.1177/1555458906289774

Frenay, M. et Bédard, D. (2004). Des dispositifs de formation universitaire s'inscrivant dans la perspective d'un apprentissage et d'un enseignement contextualisés pour favoriser la construction de connaissances et leur transfert. Dans A. Presseau et M. Frenay (dir.), Le transfert des apprentissages : comprendre pour mieux intervenir (p. 241-268). Presses de l'Université Laval. 
Fullan, M. (2015). Le leadership moteur. Comprendre les rouages du changement en éducation. Presses de l'Université du Québec.

Gélinas Proulx, A., IsaBelle, C. et Meunier, H. (2014). Compétence des nouvelles directions d'école de langue française au Canada pour la gestion inclusive de la diversité ethnoculturelle, linguistique et religieuse. Alterstice, 4(1), 73-88. http:// journal.psy.ulaval.ca/ojs/index.php/ARIRI/issue/view/8

Girard, M.-A. (2018). Diriger une école au 21e siècle. École branchée et Carrefour éducation. https://ecolebranchee.com/dossier-diriger-ecole-21e-siecle/

Grogan, M., Bredeson, P. V., Sherman, W. H., Preis, S. et Beaty, D. M. (2009). The design and delivery of leadership preparation. Dans M. D. Young, G. M. Crow, J. Murphy et R. T. Ogawa (dir.), Handbook of research on the education of school leaders (p. 395-416). Routledge.

Guertin, D. (2012). Une démarche de pratique réflexive pour la construction du savoir professionnel [Thèse de doctorat]. Université de Sherbrooke. https://www. usherbrooke.ca/pedagogie/fileadmin/sites/pedagogie/MPES-DPES/Donald Guertin.pdf

Hallinger, P. (2003). The emergence of school leadership development in an era of globalization: 1980-2002. Dans P. Hallinger (dir.), Reshaping the landscape of school leadership development: A global perspective (p. 3-22). Swets et Zeitlinger.

Hallinger, P. (2011). Leadership for leaning: Lessons from 40 years of empirical research. Journal of educational administration, 49(2), 125-142. https://doi. org/10.1108/09578231111116699

IsaBelle, C. (2017). 101 acteurs de l'éducation s'expriment : les compétences nécessaires chez les nouvelles directions d'école de langue française au Canada. Revue des sciences de l'éducation, 43(2), 15-53. https://doi.org/10.7202/1043025ar

IsaBelle, C. et Labelle, J. (2017). Rôles, responsabilités, pratiques et compétences des directions d'école. Revue des sciences de l'éducation, 43(2), 1-14. https://doi. org/10.7202/1043024ar 
IsaBelle, C., Genier, É., Davidson, A. L. et Lamothe, R. (2013). CAP : un leadership partagé entre le conseil scolaire, la direction et les enseignants. Éducation et francophonie, 41(2), 155-177. https://doi.org/10.7202/1021031ar

L'Écuyer, R. (1990). Méthodologie de l'analyse développementale de contenu. Méthode GPS et concept de soi. Presses de l'Université du Québec.

Lapointe, C. et Gauthier, M. (2005). Le rôle des directions d'écoles dans la dynamique de la réussite scolaire. Dans L. DeBlois (dir.), La réussite scolaire. Comprendre et mieux intervenir (p. 39-50). Presses de 1'Université Laval.

Le Boterf, G. (2015). Construire les compétences individuelles et collectives. Agir et réussir avec compétence, les réponses à 100 questions (7e éd.). Eyrolles.

Leurebourg, R. (2013). Rôles des directions d'école de langue française en situation minoritaire. Revue canadienne de l'éducation, 36(3), 272-297. https://journals. sfu.ca/cje/index.php/cje-rce/article/view/1548/1599

Lison, C. (2013). La pratique réflexive en enseignement supérieur : d'une approche théorique à une perspective de développement professionnel. Phronesis, 2(1), 15-27. https://doi.org/10.7202/1015636ar

Louis, K. S., Leithwood, K., Wahlstrom, K. L. et Anderson, S. E. (2010). Investigating the links to improved student learning. http://www.wallacefoundation.org/ knowledge-center/school-leadership/key-research/Documents/Investigating-theLinks-to-Improved-Student-Learning.pdf

Luc, É. et Le Saget, M. (2013). La pratique du leadership partagé : une stratégie gagnante. Presses de l'Université de Montréal.

Manning, K. (1997). Authenticity in constructivist inquiry: Methodological considerations without prescription. Qualitative Inquiry, 31(1), 93-115. https:// doi.org/10.1177/107780049700300105

Marzano, R. J., Waters, T. et McNulty, B. A. (2005). School leadership that works: From research to results. Association for Supervision et Curriculum Development.

Miles, M. B. et Huberman, A. M. (2003). Analyse des données qualitatives (M. Hlady Rispal, trad. ; 2e éd.). De Boeck. 
Ministère de l'Éducation de l'Ontario [MEO]. (2016). Compétences du 21e siècle : Phase 1 Définir les compétences du 21e siècle pour l'Ontario [Document de réflexion]. https://pedagogienumeriqueenaction.cforp.ca/wp-content/uploads/2016/02/ Ontario-21st-century-competencies-foundation-FINAL-FR_AODA EDUGAINS Feb-19 16.pdf

Mintzberg, H. (2012). Gérer tout simplement. Transcontinental.

Murphy, J. (2006). Preparing school leaders: Defining a research and action agenda. Rowman et Littlefield Education.

National Policy Board for Educational Administration. (2008). Educational leadership policy standards: ISLLC 2008. Council of Chief State School Officers. https:// www.danforth.uw.edu/uwdanforth/media/danforth/isllc-2008.pdf

Potvin, P. et Pinard, R. (2012). Deux grandes approches au Québec en prévention du décrochage scolaire : 1'approche scolaire et l'approche communautaire. Dans J.-L. Gilles, P. Potvin et C. Tièche Christinat (dir.), Les alliances éducatives pour lutter contre le décrochage scolaire (p. 129-147). Peter Lang.

Rocque, J. (2017). L'impact d'une initiative de formation internationale sur la notion de leadership et les pratiques professionnelles des directions d'école. Revue des sciences de l'éducation, 43(2), 153-189. https://doi.org/10.7202/1043029ar

Roussel, J.-F. (2011). Gérer la formation - Viser le transfert. Guérin.

Sackney, L. (2007). History of the school effectiveness and improvement movement in Canada over the past 25 years. Dans T. Townsend (dir.), International handbook of school effectiveness and improvement (p. 167-182). Springer. https://doi. org/10.1007/978-1-4020-5747-2_9

Saint-Germain, M. (2011). Le leadership et la gestion du temps. Dans J. Rocque (dir.), La direction d'école et le leadership pédagogique en milieu francophone minoritaire. Presses universitaires de St-Boniface.

Savoie-Zajc, L. (2018). La recherche qualitative/interprétative en éducation. Dans T. Karsenti et L. Savoie-Zajc (dir.), La recherche en éducation : étapes et approches (4e éd. revue et mise à jour, p. 123-150). Les Presses de l’Université de Montréal. 
Scheerens, J. (dir.). (2012). School leadership effects revisited. Review and meta-Analysis of empirical studies. Springer.

Schön, D. A. (1994). Le praticien réflexif, à la recherche du savoir caché dans l'agir professionnel (J. Heynemand et D. Gagnon, trad. et adap.). Éditions Logiques.

Tardif, J. et Meirieu, P. (1996). Stratégie pour favoriser le transfert des connaissances. Vie pédagogique, (98), 4-7.

Teddlie, C. et Stringfield, S. (2007). A history of school effectiveness and improvement research in the USA focusing on the past quarter century. Dans T. Townsend (dir.), International handbook of school effectiveness and improvement (p. 131-166). Springer.

Tournois, J., Mesnil, F. et Kop, J.-L. (2000). Autoduperie et hétéroduperie : Un instrument de mesure de la désirabilité sociale. Revue Européenne de Psychologie Appliquée, 50(1), 219-233.

Young, M. D., Crow, G., Orr, M. T., Ogawa, R. et Creighton, T. (2005). An educative look at 'educating school leaders'. UCEA Review, 47(2), 1-5. 